\title{
Enhanced deep ultraviolet luminescence from AIGaN quantum wells grown in the three-dimensional mode
}

\author{
B. Borisov, S. Nikishin, ${ }^{a)}$ V. Kuryatkov, and H. Temkin \\ Department of Electrical and Computer Engineering, Texas Tech University, Lubbock, Texas 79409
}

(Received 15 April 2005; accepted 14 September 2005; published online 31 October 2005)

\begin{abstract}
We report a significant improvement in the room temperature cathodoluminescence efficiency of $\mathrm{AlGaN}$ quantum wells when the three-dimensional growth mode is induced by reduced flux of ammonia. We interpret this observation in terms of formation of quantum dots of $\mathrm{AlGaN}$ in $\mathrm{Al}_{0.45} \mathrm{Ga}_{0.55} \mathrm{~N}$ wells. Reflection high electron diffraction images and detailed measurements of the cathodoluminescence intensity, linewidth, and wavelength as a function of growth conditions are consistent with the presence of quantum dots. (C) 2005 American Institute of Physics.
\end{abstract}

[DOI: $10.1063 / 1.2128485]$

There has been significant recent progress in achieving very short emission wavelength in light emitting diodes (LEDs) based on alloys of $\mathrm{Al}_{x} \mathrm{Ga}_{1-x} \mathrm{~N} .{ }^{1-10}$ Output powers on the order of a milliwatt, ${ }^{4,9,10}$ and good spectral purity have been obtained using a variety of growth methods and active region designs. Nevertheless, external quantum efficiencies of these devices continue to be low, on the order of $1 \%,{ }^{10}$ due to two major factors. One is the high density of threading dislocations propagating into the active region from the substrate-device interface. ${ }^{9}$ The other is poor internal quantum efficiency of wide band gap $\mathrm{Al}_{x} \mathrm{Ga}_{1-x} \mathrm{~N}$ quantum wells.

It is well known that the internal efficiency of quantum wells of InGaN is enhanced by formation of InN-rich quantum dots (QDs). ${ }^{11}$ These QDs are formed by self-assembly because of poor miscibility between $\mathrm{GaN}$ and $\mathrm{InN}$, leading to phase separation in InGaN. QDs of $\mathrm{GaN}$ can be formed by inducing a growth mode change, from two-dimensional (2D) to three-dimensional (3D), by applying $\mathrm{Si}$ anti-surfactant ${ }^{12,13}$ or by relying on residual strain in the well material. ${ }^{14,15}$ Much less is known about QDs in $\mathrm{Al}_{x} \mathrm{Ga}_{1-x} \mathrm{~N}$. Hirayama et $a l .{ }^{13}$ reported growth of $\mathrm{Al}_{x} \mathrm{Ga}_{1-x} \mathrm{~N}(0.01 \leqslant \times \leqslant 0.05)$ QDs on $\mathrm{Al}_{0.38} \mathrm{Ga}_{0.62} \mathrm{~N}$ using $\mathrm{Si}$ anti-surfactant. However, they observed decreasing luminescence efficiency with increasing AlN content in the well.

In this letter we describe a significant improvement in the room temperature cathodoluminescence (CL) efficiency of $\mathrm{Al}_{0.45} \mathrm{Ga}_{0.55} \mathrm{~N} / \mathrm{Al}_{0.55} \mathrm{Ga}_{0.45} \mathrm{~N}$ quantum wells (QWs) when the $3 \mathrm{D}$ growth mode is induced by reduced flux of ammonia in the growth of wells. Our results, interpreted in terms of QD formation in $\mathrm{Al}_{0.45} \mathrm{Ga}_{0.55} \mathrm{~N}$ wells, should result in improved quantum efficiency of deep UV LEDs.

All structures used in this study were grown by gas source molecular beam epitaxy with ammonia on (0001) sapphire substrates. Growth began with 30-min-long nitridation of sapphire, at $900{ }^{\circ} \mathrm{C}$, followed by a 150 -nm-thick AlN buffer layer grown at $\sim 860{ }^{\circ} \mathrm{C}$. Next, a 70-nm-thick undoped barrier layer of $\mathrm{Al}_{0.55} \mathrm{Ga}_{0.45} \mathrm{~N}$ was grown at $820{ }^{\circ} \mathrm{C}$. This thickness was sufficient to reach a $2 \mathrm{D}$ growth mode with $1 \times 1$ surface reconstruction, as confirmed by streaky reflection high energy electron diffraction (RHEED) patterns. The growth continued with a QW structure consisting of five

\footnotetext{
a) Author to whom correspondence should be addressed; electronic mail: sergey.a.nikishin@ttu.edu
}

pairs of $\mathrm{Al}_{0.45} \mathrm{Ga}_{0.55} \mathrm{~N}$ wells (nominally $2 \mathrm{~nm}$ thick) and $\mathrm{Al}_{0.55} \mathrm{Ga}_{0.45} \mathrm{~N}$ barriers (nominally $5 \mathrm{~nm}$ thick). QW structures were grown at temperatures between 755 and $840{ }^{\circ} \mathrm{C}$. The growth time of wells and barriers was kept the same for all structures, 20 and $50 \mathrm{~s}$, respectively. All the structures were completed with a 10-nm-thick cap layer of AlN.

The barriers were grown with an ammonia flux of 20 $\mathrm{sccm}$, corresponding to beam equivalent pressure of 1.5 $\times 10^{-5}$ Torr. All the barrier layers were grown in $2 \mathrm{D}$ growth mode, as demonstrated by the RHEED pattern of Fig. 1(a). In our previous studies, the wells and barriers were grown under the same ammonia flux of $20 \mathrm{sccm}$ and in the 2D growth mode. ${ }^{1,2,6}$ LEDs grown with this process produced $\sim 280 \mathrm{~nm}$ light with the power output of $160 \mu \mathrm{W} .{ }^{16}$ In the present study the ammonia flux used in the growth of wells was varied between 15 and $4.5 \mathrm{sccm}$, in different structures. The growth mode of the well changes with reduced flux of ammonia. For example, 2D growth mode was maintained at ammonia fluxes greater than $7 \mathrm{sccm}$, at a substrate temperature of $795^{\circ} \mathrm{C}$. At the ammonia flux of $6.5 \mathrm{sccm}$ we start to observe spots in the RHEED pattern, as shown in Fig. 1(b).

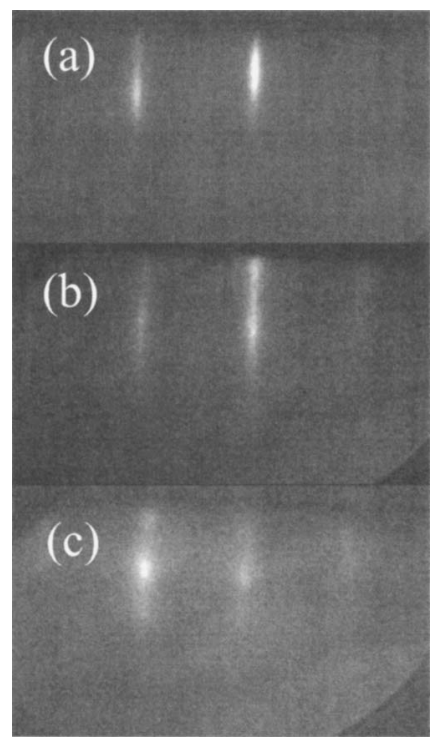

FIG. 1. Variation of the RHEED patterns during the growth. (a) Twodimensional grown barrier at $20 \mathrm{sccm}$ of ammonia, (b) 2D-3D grown well at $6.5 \mathrm{sccm}$ of ammonia, (c) 3D grown well at $5.5 \mathrm{sccm}$ of ammonia. 


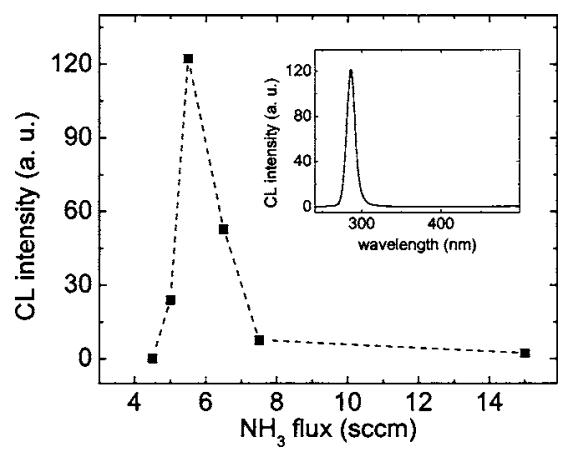

FIG. 2. Room temperature CL intensity of $\mathrm{Al}_{0.45} \mathrm{Ga}_{0.55} \mathrm{~N} / \mathrm{Al}_{0.55} \mathrm{Ga}_{0.45} \mathrm{~N}$ QWs vs an ammonia flux. The inset shows CL spectrum of QW grown in 3D growth mode at optimal flux of ammonia of $5.5 \mathrm{sccm}$.

With the ammonia flux reduced to $5.5 \mathrm{sccm}$ the RHEED patterns become quite spotty. This behavior of the RHEED pattern is typical of growth mode change from $2 \mathrm{D}$ to $3 \mathrm{D} .{ }^{17}$ Well growth could not be continued for ammonia fluxes below $4.5 \mathrm{sccm}$. It should be noted that for all ammonia fluxes used for well growth the RHEED pattern of the barrier layer recovers and shows $2 \mathrm{D}$ growth by the time the next well is grown. In other words, the barriers are sufficiently thick for the growth to planarize the barrier surface before the next well is nucleated. This implies that any $3 \mathrm{D}$ grown islands are confined to individual wells. While the barrier-well interfaces are flat, the well-barrier interfaces are rough.

Transition from the 2D to 3D mode, induced by reduced flux of ammonia, has a dramatic effect on the CL intensity. Figure 2 shows the evolution of the CL intensity as a function of the flux of ammonia for a set of QW structures grown at $795^{\circ} \mathrm{C}$. A maximum CL intensity was obtained at ammonia flux of $\approx 5.5 \mathrm{sccm}$. This should be compared to the CL of the same structure grown under ammonia flux leading to $2 \mathrm{D}$ growth, i.e., greater than $7 \mathrm{sccm}$. The CL intensity at low ammonia flux is enhanced by a factor greater than 10 . The CL spectrum, shown in the inset of Fig. 2, shows only emission at $\sim 280 \mathrm{~nm}$. Emission from the barriers is not visible, indicating excellent carrier capture efficiency, and we do not observe any deep level emission. The peak in CL intensity is quite narrow, even a small change of $\pm 0.5 \mathrm{sccm}$ in the flux of ammonia results in significant reduction in the CL emission. Similar dependence on the ammonia flux is observed for structures grown at different temperatures, as discussed below.

A detailed dependence of the CL emission intensity, wavelength, and linewidth on the growth temperature is illustrated in Fig. 3 for two fluxes of ammonia. With the ammonia flux set at $5.5 \mathrm{sccm}$, resulting in a 3D growth mode, the CL intensity reaches a maximum at the growth temperature of $795^{\circ} \mathrm{C}$, as illustrated in Fig. 3(a). The intensity decreases by a factor of 2 for temperature deviation of $\pm 15^{\circ} \mathrm{C}$. With the ammonia flux fixed at $6.5 \mathrm{sccm}$, corresponding to a transition between 2D and 3D growth modes, the CL intensity peaks within a narrower range of temperatures, less than $\pm 10^{\circ} \mathrm{C}$, centered on $810^{\circ} \mathrm{C}$. While the intensity dependence on the growth temperature is qualitatively similar for the two fluxes of ammonia, the CL wavelength dependence is very different. Structures grown with the ammonia flux of 6.5 sccm exhibit CL emission close to $280 \mathrm{~nm}$ and weak wavelength dependence on the growth temperature. This suggests that there is no change in either the well thickness or com-
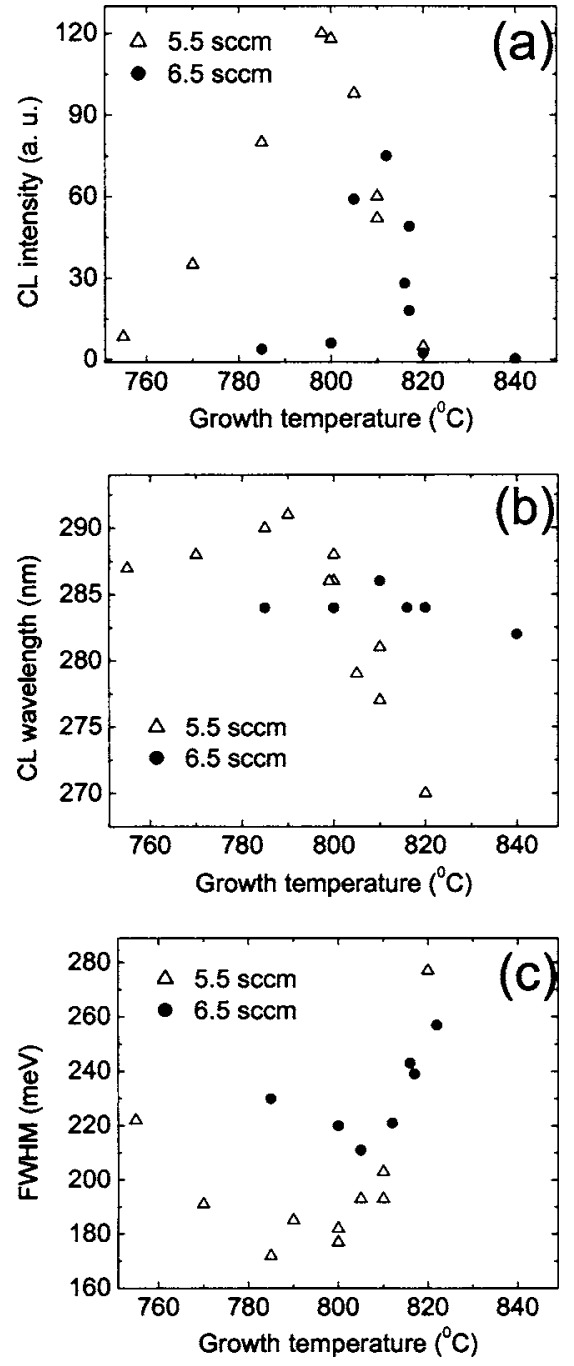

FIG. 3. Room temperature CL peak intensity (a), position (b), and FWHM (c) vs growth temperature.

position. In contrast, structures grown with $5.5 \mathrm{sccm}$ show a weak redshift of $\sim 4 \mathrm{~nm}$ for low growth temperatures and a pronounced blueshift of $\sim 20 \mathrm{~nm}$ at high temperatures. While the slight redshift may be due to the slight increase in the well thickness, the origin of the blueshift is more complicated. A blueshift could be produced by a decrease in the well thickness due to evaporation of $\mathrm{Ga}$ at high temperatures and the resulting increase in the effective AlN content in the well. A calculation of the blueshift in the emission wavelength shows that the well thickness would have to be reduced from $2.4 \mathrm{~nm}$ at $795{ }^{\circ} \mathrm{C}$ to $0.8 \mathrm{~nm}$ at $820{ }^{\circ} \mathrm{C}$, assuming no change in composition. Such a change in thickness is inconsistent with our growth rate-growth temperature data for thick $\mathrm{Al}_{\mathrm{x}} \mathrm{Ga}_{1-\mathrm{x}} \mathrm{N}$ with $0.45 \leqslant \times \leqslant 0.55$. We also know that the intentional reduction in the well thickness, without the change in the growth mode, does not result in an increase in the CL efficiency, despite increased overlap between electron and hole wave functions. ${ }^{18}$ Furthermore, an increase in the average AlN content in the well cannot occur without a change in the growth rate, since this could happen only by the evaporation of Ga. The observed blueshift can be accounted for by formation of 3D structures in the wells, quantum dots. Formation of quantum dots is known to result in a blueshift, the magnitude of which depends on their size and composition. ${ }^{19}$ 
Another interesting aspect of the optical properties of QWs grown in the 3D mode is the reduced linewidth of the CL emission. Figure 3(c) shows the temperature dependence of the CL linewidth, full width at half maximum (FWHM), of two sets of samples. The lowest linewidth of 170-175 $\mathrm{meV}$ is reached in samples grown in the 3D mode at 5.5 sccm of ammonia and the growth temperature of 785$795^{\circ} \mathrm{C}$. This is the temperature corresponding to the highest CL emission intensity. This type of dependence on growth temperature, with a clear minimum in FWHM and a corresponding maximum in the intensity, is never observed for ammonia fluxes greater than $6.5 \mathrm{sccm}$ or lower than 5.5 $\mathrm{sccm}$. The CL linewidth of structures grown under mixed 2D-3D conditions is larger and less sensitive to growth temperature, as illustrated in Fig. 3(c). Lower temperature sensitivity of structures grown in mixed 3D-2D mode would imply the onset of quantum dot formation. The linewidth reduction in structures grown in the $3 \mathrm{D}$ mode cannot be attributed to flatter interfaces between wells and barriers since our RHEED data show that the well-barrier interface is rough. Another possibility might be formation of inversion domains resulting in increased CL intensity and reduced linewidth. ${ }^{20,21}$ However, formation of such domains should result in a $3 \times 3$ or $6 \times 6$ surface reconstruction at low temperatures, that could be observed in RHEED pattern upon wafer cooldown to $\sim 300{ }^{\circ} \mathrm{C}^{21}$ This possibility can be excluded since only a $2 \times 2$ reconstruction, corresponding to single domain layers, is observed in low-temperature RHEED images of our structures. On the other hand, the size of QDs, their size distribution, and density are determined by trade-offs between growth temperature and surface kinetics and the observed dependence could be explained in these terms. While the formation of QDs is consistent with our data, more structural information about QD ensemble size and size distribution are needed to interpret the $\mathrm{CL}$ wavelength/linewidth, if not considering quantized states of QD structures. The atomic microscope studies of bare dots are under way right now. We are also studying time resolved photoluminescence of these structures to determine the correlation with the growth trend. However, irrespective of the mechanism, the CL enhancement observed for growth at low $\mathrm{III} / \mathrm{V}$ ratios is very significant and of interest in UV light sources.

In summary, we observe a significant increase in the 280 nm CL emission from QW structures of AlGaN upon transition to 3D growth. We interpret this observation in terms of formation of quantum dots of $\mathrm{AlGaN}$. This is supported by RHEED images and detailed measurements of the CL inten- sity, linewidth, and wavelength as a function of growth conditions.

This study was supported by NSF [ECS-0323640 and ECS-0304224], DARPA, under SUVOS program monitored by Dr. J. Carrano, and the J. F Maddox Foundation.

${ }^{1}$ G. Kipshidze, V. Kuryatkov, B. Borisov, S. Nikishin, M. Holtz, S. N. G. Chu, and H. Temkin, Phys. Status Solidi A 192, 286 (2002).

${ }^{2}$ G. Kipshidze, V. Kuryatkov, K. Zhu, B. Borisov, M. Holtz, S. Nikishin, and H. Temkin, J. Appl. Phys. 93, 1363 (2003).

${ }^{3}$ V. Adivarahan, S. Wu, A. Chitnis, R. Pachipulusu, V. Mandavilli, M. Shatalov, J. P. Zhang, M. A. Khan, G. Tamulaitis, A. Sereika, I. Yilmaz, M. S. Shur, and R. Gaska, Appl. Phys. Lett. 81, 3666 (2002).

${ }^{4}$ J. P. Zhang, S. Wu, A. Rai, V. Mandavilli, V. Adivarahan, A. Chitnis, M. Shatalov, and M. A. Khan, Appl. Phys. Lett. 83, 3456 (2003), and references therein.

${ }^{5}$ A. Yasan, R. McClintock, K. Mayes, D. Shiell, L. Gautero, S. R. Darvish, P. Kung, and M. Razeghi, Appl. Phys. Lett. 83, 4701 (2003).

${ }^{6}$ S. A. Nikishin, V. V. Kuryatkov, A. Chandolu, B. A. Borisov, G. D. Kipshidze, I. Ahmad, M. Holtz, and H. Temkin, Jpn. J. Appl. Phys., Part 2 42, L1362 (2003).

${ }^{7}$ A. Hanlon, P. M. Pattison, J. F. Kaeding, R. Sharma, P. Fini, and S. Nakamura, Jpn. J. Appl. Phys., Part 2 42, L628 (2003).

${ }^{8}$ K. Mayes, A. Yasan, R. McClintock, D. Shiell, S. R. Darvish, P. Kung, and M. Razeghi, Appl. Phys. Lett. 84, 1046 (2004).

${ }^{9}$ A. J. Fischer, A. A. Allerman, M. H. Crawford, K. H. A. Bogart, S. R. Lee, R. J. Kaplar, W. W. Chow, S. R. Kurtz, K. W. Fullmer, and J. J. Figiel, Appl. Phys. Lett. 84, 3394 (2004).

${ }^{10}$ V. Adivarahan, S. Wu, J. P. Zhang, A. Chitnis, M. Shatalov, V. Mandavilli, R. Gaska, and M. A. Khan, Appl. Phys. Lett. 84, 4762 (2004).

${ }^{11}$ S. Nakamura and G. Fasol, The Blue Laser Diode (Springer, Berlin, 1997).

${ }^{12}$ S. Tanaka, S. Iwai, and Y. Aoyagi, Appl. Phys. Lett. 69, 4096 (1996).

${ }^{13}$ H. Hirayama, Y. Aoyagi, and S. Tanaka, MRS Internet J. Nitride Semicond. Res. 4S1, G9.4 (1999).

${ }^{14}$ B. Daudin, F. Widmann, G. Feuillet, Y. Samson, M. Arlery, and J. L. Rouviere, Phys. Rev. B 56, R7069 (1997).

${ }^{15}$ F. Widmann, B. Daudin, G. Feuillet, Y. Samson, J. L. Rouvie're, and N. Pelekanos, J. Appl. Phys. 83, 7618 (1998).

${ }^{16}$ S. Nikishin, M. Holtz, and H. Temkin, Jpn. J. Appl. Phys., Part 1 44, 7221 (2005).

${ }^{17}$ See, for example, M. B. Panish and H. Temkin, Gas Source Molecular Beam Epitaxy (Springer, Berlin, 1993).

${ }^{18}$ B. Borisov, V. Kuryatkov, S. Nikishin, H. Temkin, K. A. Bulashevich, I. A. Zhmakin, V. F. Mymrin, and S. Yu. Karpov (unpublished).

${ }^{19}$ D. Huang, Y. Fu, and H. Morkoç, in Optoelectronic Properties of Semiconductor Nanostructures, edited by T. Steiner (Artech House, Boston, 2004).

${ }^{20}$ P. J. Schuck, M. D. Mason, R. D. Grober, O. Ambacher, A. P. Lima, C. Miskys, R. Dimitrov, and M. Stutzmann, Appl. Phys. Lett. 79, 952 (2001).

${ }^{21}$ T. V. Shubina, V. N. Jmerik, M. G. Tkachman, V. A. Vekshin, V. V. Ratnikov, A. A. Toropov, A. A. Sitnikova, S. V. Ivanov, J. P. Bergman, F. Karlsson, P. Holtz, and B. Monemar, Phys. Status Solidi B 234, 919 (2002). 